\title{
Proteomic Investigation of Changes in Rat Skeletal Muscle after Exercise-Induced Fatigue
}

\author{
Liping-zhao ${ }^{1 \#}$, Wenhui-Yan ${ }^{2 \#}$, Heng-Xiang ${ }^{1}$, Xiaoyang-wang ${ }^{3 *}$, Haixuan-qiao ${ }^{2 *}$ \\ ${ }^{1}$ Department of Rehabilitation and sports medicine, Tianjin Medical University, Tianjin, China \\ 2 School of biomedical engineering, Tianjin Medical University, Tianjin 300070, China (E-mail: qiaohaixuan@yahoo.com.cn) \\ ${ }^{3}$ Department of Physical Education, Shanxi University, Shanxi, Taiyuan 030006, (E-mail:wangxy1212@163.com)
}

\begin{abstract}
The mechanisms of exercise-induced fatigue have not been investigated using proteomic techniques, an approach that could improve our understanding and generate novel information regarding the effects of exercise. In this study, the proteom alterations of rat skeletal muscle were investigated during exercise-induced fatigue. The proteins were extracted from the skeletal muscle of SD rat thigh, and then analyzed by two-dimensional electrophoresis and PDQuest software. Compared to control samples, 10 significantly altered proteins were found in exercise samples, two of them were upregulated and eight of them were downregulated. These proteins were identified by MALDI TOF-MS. The two upregulated proteins were identified as MLC1 and myosin L2 (DTNB) regulatory light-chain precursors. The eight decreased proteins are Glyceraldehyde-3-phosphate Dehydrogenas (GAPDH); Beta enolase; Creatine kinase M chain (M-CK); ATPAMP Transphosphorylase (AK1); myosin heavy chain (MHC); actin; Troponin I, fast-skeletal muscle (Troponin I fast-twitch isoform), fsTnI; Troponin T, fast-skeletal muscle isoforms (TnTF). In these proteins, four of the eight decreased proteins are related directly or indirectly to exercise induced fatigue. The other proteins represent diverse sets of proteins including enzymyes related to energy metabolism, skeletal muscle fabric protein and protein with unknown functions. They did not exhibit evident relationship with exercise-induced fatigue. Whereas the two identified increased proteins exhibit evident relationship with fatigue. These findings will help in understanding the mechanisms involved in exercise-induced fatigue.
\end{abstract}

Key words: Proteomics, exercise-induced fatigue, skeletal muscle, rat

\section{INTRODUCTION}

Exercise-induced fatigue is a reduction in maximal voluntary muscle force that results from intense physical activity (Gandevia. S. C. 2001). Exercise-induced fatigue is a complex process. In the course of exercise the physiological environment changes, which could result in the alteration of gene and protein expression levels and the alteration of posttranslational modifications. Importantly, intense and prolonged exercise can result in changes in cell signaling pathways in eukaryotic cells (Reid MB. 2008; Powers SK et al. 2008). Thus, the proteome profile of skeletal muscles during exercise-induced fatigue is distinct from that of the normal physiological state. Although many proteins associated with exercise have been identified (Adam J et al. 2009; Borja Guerra et al, 2010; Craig A. Goodman et al. 2009), the molecular mechanisms involved in exercise-induced fatigue have not been well elucidated. Investigation into the alterations of proteins in exercise-induced fatigue will help to discover mechanisms, pathways and new players involved in the induction and regulation of fatigue.

Proteomics is the study of protein properties (expression level, posttranslational modification, interactions, etc.) on a large scale to obtain a more global view of physiological processes. The application of proteomics to investigate exercise is in its infancy (D.S. Hittel et al. 2007), and few studies have been published in this area (Jatin G. Burniston 2008; Yamaguchi
W et al. 2010). in order to understand the mechanisms of exercise-induced fatigue and search for new proteins involved in it. In the present study, an exercise-induced fatigue model of rat SD was built using swimming. Biochemical and behavioral indices were used to verify the credibility of the model. After the modeling, the skeletal muscle of rat SD thigh was separated at the low temperature condition and the proteins were extracted.

Proteins of skeletal muscle were separated by twodimensional (2D) gel electrophoresis. The protein patterns of scanned 2D images were analyzed with PDQuest software and 10 proteins were found to be upregulated or downregulated. These altered proteins were then identified by MALDI TOFMS/MS and searched against the SWISS-PROT and NCBInr database. Our findings reveal novel changes in the expression of well-known metabolic enzymes and myofibrillar proteins. Some of these are related to fatigue, while others have not been confirmed as involved in the process of fatigue. Therefore, further investigation of their roles during fatigue will help to elucidate the mechanism of exercise induced fatigue.

\section{MATERIALS AND METHODS}

The model of exercise-induced fatigue

The model of exercise-induced fatigue was established as described by (Shin Terada. 2001, HOU L. 2005). Nine- to

Note: Liping-zhao and Wenhui-Yan contributed equally to this paper.

*Corresponding author: Dr. Haixuan-Qiao. School of biomedical engineering, Tianjin Medical University, Tianjin 300070, China (E-mail: qiaohaixuan@yahoo.com.cn) or Xiao Yang-Wang Departament of Physical Education, Shanxi University, Shanxi, Taiyuan 030006, China (E-mail: Wangxy122@163.com)

Received: December 22, 2010. In revised form: May 26, 2011. Accepted: June 15, 2011 
ten-week-old male Sprague-Dawley rats with initial body weights of $200 \pm 20 \mathrm{~g}$ were used for this study. Sixteen male SD rats were randomly divided into exercise and rest groups according to weight. In the exercise group, the rats repeated 30-minute swimming bouts daily for the first 2 days. This was increased by $30 \mathrm{~min}$ each day for the next 3 days, reaching 120 minutes of swimming by the fifth day. This was increased to 180 minutes for the sixth and seventh days. For the final three days the exercise was doubled by introduced 180-minute swimming bouts interspaced with six hours for rest. (Table 1). All rats swam in a barrel filled to a depth of $80 \mathrm{~cm}$ and an average surface area of $190 \mathrm{~cm} 2 /$ rat. Water temperature was maintained at $35^{\circ} \mathrm{C}$ during swimming training. Changes in body weight and the biochemical index blood urea nitrogen (BUN) were used to verify the credibility of the model.

\section{Preparation of skeletal muscle proteins}

The rats from the exercise and control groups were killed by decollation, proteins were extracted from the skeletal muscle of SD rat thigh at a low temperature and dissolved in $9 \mathrm{~mol} / \mathrm{L}$ urea, 2\% CHAPS ( Sigma), Pharmalyte $\mathrm{pH}$ 3-10 (Amersham Pharmacia), 1\% DTT, and then separated from insoluble material by centrifugation. The total protein concentration was determined by the Bradford assay (Kruger N J et al. 1996).

2-Dimensional gel electrophoresis and image analysis

Two-dimensional gel electrophoresis (2DE) was carried out in the IPGphor system (BioRad) as described (Gorg et

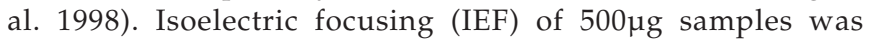
performed in pre-cast immobilized $\mathrm{pH}$ gradient (IPG) strips ( $\mathrm{pH} 3-10, \mathrm{~L}=18 \mathrm{~cm}$; Pharmacia) using the following rehydration and running conditions: rehydration 14hours, step 1: $250 \mathrm{~V}, 30 \mathrm{~min}$, step 2: $1000 \mathrm{~V}, 1$ hour, step3: $1000-10000 \mathrm{~V}$, 5-6hours, step 4: 10 000V, 60 000Vh. After IEF separation, the extruded strips were incubated in buffer $1(50 \mathrm{mmol}$ Tris-HCl, pH 8.18, 6 mol urea, $30 \%$ glycerol, $2 \%$ SDS, 015 $\%$ DTT) and buffer $2(50 \mathrm{mmol} / \mathrm{L}$ Tris- $\mathrm{HCl}, \mathrm{pH} 8.18,6 \mathrm{~mol} / \mathrm{L}$ urea, 30\% glycerol, $2 \%$ SDS, $4.5 \%$ iodoacetamide) for $15 \mathrm{~min}$, respectively. The equilibrated gels were immediately applied to $12 \%$ polyacrylamide slab gels for second separation. Gel electrophoresis was carried out at a constant current $(30 \mathrm{~mA} /$ gel) and a set temperature of $20^{\circ} \mathrm{C}$. Following electrophoresis, the gels were stained with Coomassie Brilliant Blue. Analysis of the gels, including background subtraction, spot detection, volume normalization and differences in protein expression levels among samples were analyzed by using PDQuest software (version 6.0, BioRad), To determine the variation, 3 gels were prepared for each sample. The protein spots that varied $>2$ fold changes and were specific for the test groups and the control group were manually labeled and considered for MS analysis.

\section{In-gel digestion and mass spectrometric identification}

Gel spots were excised from the stained gel, and cut to $1-2 \mathrm{~mm}^{2}$ slices. The excised pieces were destained with 25 mmol ammonium bicarbonate/50 \% acetonitrile (Fisher), and were washed with $100 \%$ acetonitrile, and then dried with a SpeedVac Plus SC110A vacuum concentrator (Savant, Holbook, NY, USA). Trypsin in digestion buffer (25 mmol ammonium bicarbonate P5 mmolPL calcium chloride) was added to the dry gel pieces and incubated for 15 hours for rehydration at $37^{\circ} \mathrm{C}$. The resulting peptide mixture was extracted with $5 \%$ TFA for 1 hour at $40^{\circ} \mathrm{C}$ and followed by $2.5 \%$ TFA $/ 50 \%$ acetonitrile for 1 hour at $30^{\circ} \mathrm{C}$. The supernatants were pooled and the peptides were dried by a vacuum concentrator. Peptide sequences were identified using matrix assisted laser desorption Pionization time of flight mass spectrometry (MALDI-TOF MS). Prior to mass spectrometry, the peptide samples were reconstituted with $0.5 \%$ TFA. Peptide mass mapping of tryptic digests was carried out with a Micromass Tof Spec MALDI-TOF mass spectrometer. The datasets of the MS spectra, including peptide sequence information, were searched against the SWISS-PROT (GeneBio, Geneva, Switzerland) and NCBInr database using Mascot Daemon (Matrix Science, London, UK) as a client attached to the Mascot search protocol.

Immunoblot analysis

The proteins were extracted from the skeletal muscle of SD rat thigh and lysed in a buffer $(0.5 \mathrm{M}$ Tris $\mathrm{HCl}, 0.4 \%$ SDS, $20 \%$ glycerol, $1.5 \%$ BromphenolBlue, $1.0 \%$ beta-mercap toethanol) by boiling for $5 \mathrm{~min}$. Equal amounts of protein were

TABLE 1

Sswimming periods per day over the ten day experiment

\begin{tabular}{ll}
\hline & SWIMMING PERIODS \\
\hline First day & $30 \mathrm{~min}$ \\
Second day & $30 \mathrm{~min}$ \\
Third day & $60 \mathrm{~min}$ \\
Fourth day & $90 \mathrm{~min}$ \\
Fifth day & $120 \mathrm{~min}$ \\
Sixth day & $180 \mathrm{~min}$ \\
Seventh day & $180 \mathrm{~min}$ \\
Eighth day & $180 \mathrm{~min}$, two times, The interval between the two times measures 6 hours \\
Ninth day & $180 \mathrm{~min}$, two times, The interval between the two times measures 6 hours \\
Tenth day & $180 \mathrm{~min}$, two times, The interval between the two times measures 6 hours, \\
\hline
\end{tabular}


subjected to $12 \%$ SDS gel electrophoresis and transferred to polyvinylidenediuoride membranes (AmershamBiosciences, Piscataway, NJ, USA). Membranes were probed with antibodies as indicated below and detected using ECL Advance or Plus (Amer-sham Biosciences). The blots were probed with the following antibodies, Rabbit anti-Actin (1:1000, cell signal), Rabbit anti-alpha Tubulin (1:1000), anti-rabbit HRP-conjugated secondary antibody (1:5000), and polyclonal anti-GAPDH antibody (1:500) (Santa Cruz, CA, USA).

\section{Statistic analysis}

Protein expression data for control and treated groups are expressed as the mean \pm SD of 3 replicate gels for fold changes of normalized spot volumes. For the statistic analysis of data, the Student-t-test was used, with $\mathrm{p}<0.05$ considered as significant.

\section{RESULT}

Effect of swimming on body weight and BUN of rats

After ten days of loading-increasing swimming exercise, the body weight of the exercise group had decreased significantly and the BUN had increased significantly compared to the control groups (Fig 1, Fig 2). These changes of behavioral

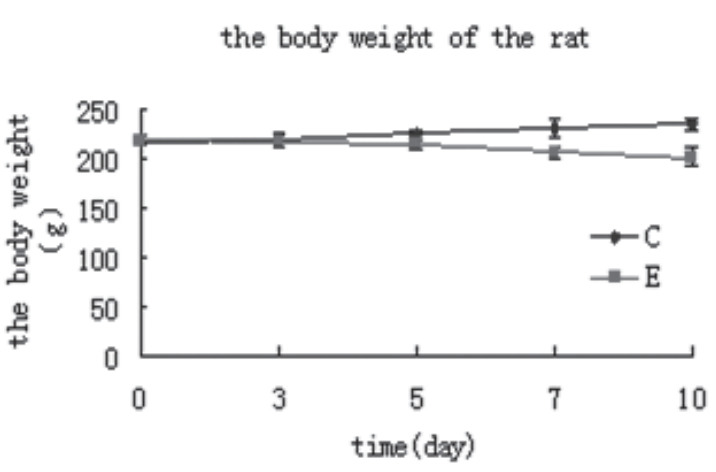

Fig. 1: The changes of the rat body weight in the course of exercise (C: contral group, E exercise group)

the FUI of the rat

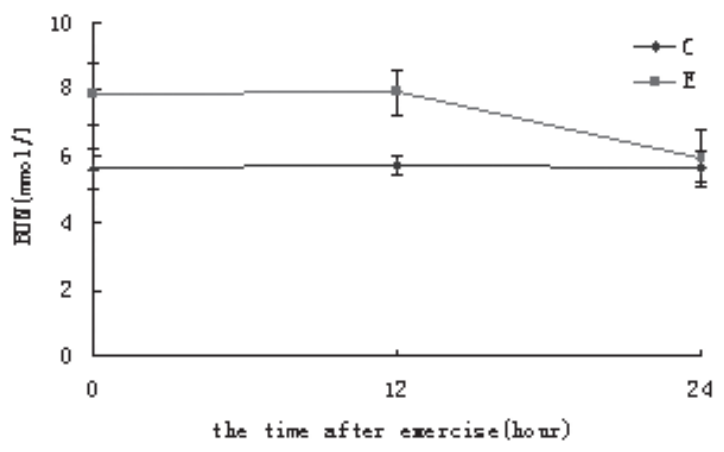

Fig. 2: The changes of the BUN concentration of rat after exercise (C: contral group, E exercise group)

and biochemical index showed that the rats were in the condition of fatigue. The model was suitable for the use of the experiments about exercise-induced fatigue.

\section{Protein expression profile}

To investigate the potential proteomic alterations of rat skeletal muscle during exercise-induced fatigue, the proteins were extracted from the skeletal muscle of SD rat thigh and separated by two dimensional gel electrophoresis, The muscles were stained with Coomassie Brilliant Blue and the data was analyzed by PDQuest software (Fig 3).

Figure 3 shows a pair of Coomassie Brilliant Blue stained gels obtained from rat skeletal muscle proteins during exercise-induced fatigue. The distributing modes of overall skeletal muscle proteins were very similar to each other, the range of pI was 4.0-9.0, and the Mr range was 20-100 kD. The overall protein profiles in the control and exercise groups were compared by image analysis. Using $\mathrm{pH} 3-10$ stripes, computer assisted analysis of the gels detected about 400 spots. Compared to control samples, ten proteins were apparently upregulated or downregulated in treated rats, determined by two dimensional gel electrophoresis and PDQuest software analysis. These proteins exhibited $>2$ fold changes between the exercise and control group. Two of them were upregulated and eight of them were down regulated.
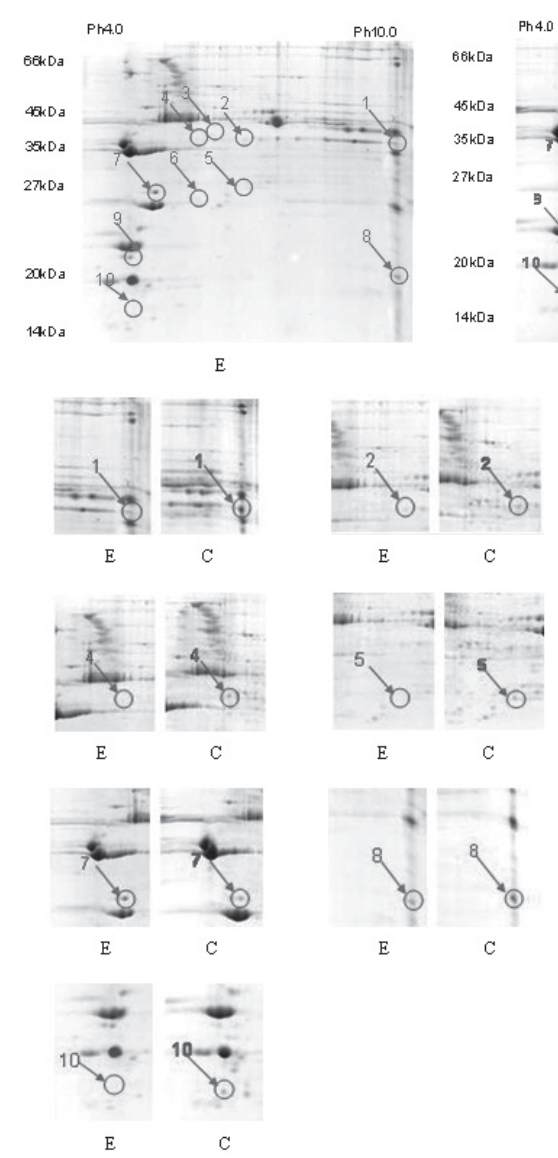

E

C

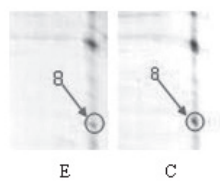

E
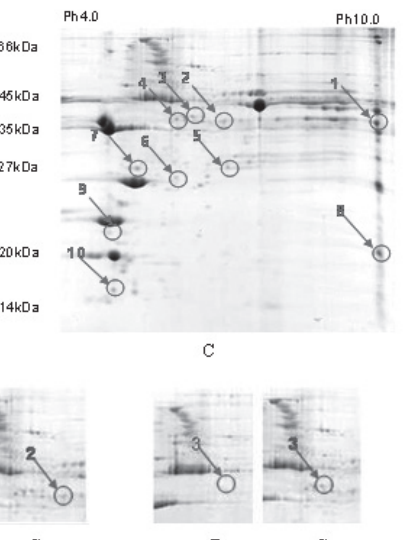

C

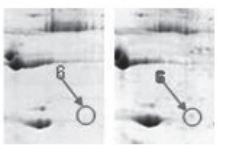

E C

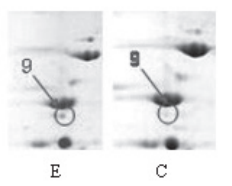

Fig. 3: 2-D map of SD rat thigh (E: exercise group, $C$ : control groups), number 1 to number 10 indicate the up-regulated or down-regulated proteins in exercise group. 
These proteins were identified by MALDI-TOF MS and the Mascot search program. Two of the upregulated proteins were identified as myosin L2 (DTNB) regulatory light chain precursor ( $\mathrm{pI}$ 4.82, Mr 19kD), and Myosin light chain 1 (MLC1, pI 5.03, Mr 22kD).

The eight downregulated proteins were identified as Beta enolase (pI 7.74, Mr 46.8 kD); Glyceraldehyde-3-phosphate Dehydrogenas (GAPDH, pI 8.44, Mr 35.8kD); Creatine kinase $\mathrm{M}$ chain (M-CK, pI 6.58, Mr 43kD); Adenylate kinase isoenzyme 1, ATP-AMP Transphosphorylase (AK1, pI 7.71, Mr 21.6 kD); Myokinase; myosin heavy chain (MHC, pI 5.31, Mr 24 kD); actin (pI 5.23, Mr $42 \mathrm{kD}$ ); Troponin I, fast skeletal muscle (fsTnI, Troponin I, fast-twitch isoform, pI 8.86, Mr $21.2 \mathrm{kD}$ ); Troponin $\mathrm{T}$, fast skeletal muscle isoforms (TnTF, pI 6.18, Mr 30.6kD).

The four downregulated proteins (GAPDH; Beta enolase; M-CK; ATP-AMP Transphosphorylase) are enzymes related to energy metabolism; the other four downregulated proteins, MHC; actin; fsTnI; TnTF) are muscle fabric proteins (Table 2, Table 3).

Immunoblot verification of Actin and GAPDH

Downregulation of actin and GAPDH expression was found in the muscles of the exercise group by 2-DE. Western blot analysis was then performed for these two proteins in an independent set of skeletal muscle samples. Western blot showed the consistent downregulation of actin and GAPDH in the exercise group compared to the control group, Levels of these two proteins were significantly lower in the muscle of the exercise sample compared to controls (Fig 4).

\section{DISCUSSION}

During intense activation of skeletal muscle, contractile function becomes impaired. This is known as fatigue. The cause of fatigue is probably multifactorial, but a central role is changes in highenergy phosphates (i.e. ATP and ADP). The accumulation of by-products of rapid energy metabolism has also been postulated (Westerblad $\mathrm{H}$ et al. 1998). Therefore, the proteome profile of skeletal muscle during exercise-induced fatigue is distinct from that of the normal physiological state. In this study, the alterations of skeletal muscle proteins during exercise-induced fatigue were analyzed by two-dimensional electrophoresis. The results showed that 10 proteins were apparently upregulated or downregulated after exercise-induced fatigue. Two of them were upregulated and eight were downregulated.

In these proteins, four of the eight decreased proteins may relate to exercise induced fatigue directly or indirectly. The

TABLE 2

Identification of differentially expressed protein spots in treated rats by mass spectrometry and database searches

\begin{tabular}{lllllll}
\hline $\begin{array}{c}\text { Spots } \\
\text { number }\end{array}$ & $\begin{array}{c}\text { SWISS-PROT } \\
\text { ID }\end{array}$ & $\begin{array}{c}\text { Theoretical } \\
\text { pI }\end{array}$ & $\begin{array}{c}\text { Theoretical } \\
\text { Mr(kDa) }\end{array}$ & $\begin{array}{c}\text { Sequence } \\
\text { covered }\end{array}$ & $\begin{array}{c}\text { MASquot } \\
\text { Score }\end{array}$ & \multicolumn{1}{c}{ Protein name } \\
\hline 1 & XP217111.1 & 8.44 & 35.8 & $14 \%$ & 275 & $\begin{array}{l}\text { glyceraldehyde-3-phosphate } \\
\text { dehydrogenase } \\
\text { beta enolase }\end{array}$ \\
2 & XP229872.1 & 7.62 & 46.2 & $8 \%$ & 199 & creatine kinase, M chain \\
3 & P00564 & 6.58 & 43.0 & $8 \%$ & 80 & Actin \\
4 & ATRT & 5.23 & 42.0 & $10 \%$ & 154 & ATP-AMP transphosphorylase \\
5 & P39069 & 7.71 & 21.6 & $17 \%$ & 133 & troponinT, fast skeletal muscle isoforms \\
6 & P09739 & 6.18 & 30.6 & $6 \%$ & 33 & myosin light chain 1 \\
7 & P16409 & 5.03 & 22.0 & $22 \%$ & 203 & troponin I, fast-twitch isoform \\
8 & P27768 & 8.86 & 21.2 & $16 \%$ & 161 & myosin L2 (DTNB) regulatory light chain precursor \\
9 & MORTL2 & 4.82 & 19.0 & $5 \%$ & 85 & myosin heavy chain \\
10 & AAA41656.1 & 5.31 & 24.0 & $5 \%$ & 45 & \\
\hline
\end{tabular}

TABLE 3

Fold changes of differentially expressed proteins in treated group compared to untreated group $(p<0.05)$

\begin{tabular}{clc}
\hline $\begin{array}{c}\text { Spot } \\
\text { no }\end{array}$ & Protein name & Fold changes \\
\hline 1 & glyceraldehyde-3-phosphate dehydrogenase (GAPDH) & -4.87 \\
2 & beta enolase & -2.05 \\
3 & creatine kinase, M chain (M-CK) & -2.14 \\
4 & Actin & -2.27 \\
5 & ATP-AMP transphosphorylase & -2.16 \\
6 & troponinT, fast skeletal muscle isoforms (TnTF) & -1.93 \\
7 & myosin light chain 1(MLC1) & +2.73 \\
8 & troponin I, fast-twitch isoform (fsTnI) & -2.68 \\
9 & myosin L2 (DTNB) regulatory light chain precursor & +2.58 \\
10 & myosin heavy chain (MHC) & -2.47 \\
\hline
\end{tabular}

Data represents mean \pm SD of 3 experiments. (+) indicates upregulation, whereas $(-)$ indicates downregulation 
other proteins represent diverse sets of proteins including enzyme related to energy metabolism, skeletal muscle protein that do not exhibit evident relationship with exercise.induced fatigue. Whereas, the two increased proteins that were identified exhibit evident relationships with fatigue.

GAPDH is a classical glycolytic protein in energy production (Anders J et al, 2000). However, recent evidence has demonstrated that mammalian GAPGH displays a number of diverse activities unrelated to its glycolytic function. These include its role in membrane fusion, RNA transport, microtubule assembly, DNA replication, DNA repair, and apoptosis (Chuang DM et al. 2005). Exercise promotes consumption of energy sources such as glycogen by mobilizing internal energy metabolism to the maximum and using and depleting the energy source. The role of GAPDH in fatigue may correlate with its energy supply for movement and transport.

Creatine kinase $(\mathrm{CK})$ is a key enzyme for maintaining a constant ATP/ADP ratio during rapid energy turnover. It catalyzes phosphate exchange between the high free energy phosphates ATP and phosphocreatine $(\mathrm{PCr})$ via the reaction: $\mathrm{PCr}+\mathrm{ADP}+\mathrm{H}+\leftrightharpoons$ creatine $(\mathrm{Cr})+\mathrm{ATP}$. In skeletal muscle there are two major forms of CK: one is found in the cytosol (M-CK) and the other is associated with mitochondria (ScCKmit). M-CK dominates in fast-twitch muscles and is considered important for energy utilization at sites of high energy turnover (LaBella J J et al. 1998), Several studies have demonstrated that the complete absence of CK activity is associated with an immediate marked decline in skeletal muscle force generation and power output during repetitive activation (Steeghs K A et al.1997; Anders J et al. 2000;
Watchko J F et al.1997; Momken I et al.2005), Our result is consistent with these previous findings.

Beta enolase is a glycolytic enzyme of particular interest because it has muscle specific isoforms (Chosa $\mathrm{E}$ et al. 2003). In adults, subunits of the muscle isoform of enolase (b-enolase) accumulate preferentially in fast-twitch muscle fibers in which the b-isoenzyme accounts for more than $90 \%$ of total enolase. In this study, Beta enolase was found to have decreased in skeletal muscle. However, its role in fatigue remain unclear and deserves further investigation.

Actin is a globular, roughly $42-\mathrm{kDa}$ protein found in all eukaryotic cells. Actin participates in many important cellular processes including muscle contraction, cell motility, cell division and cytokinesis, vesicle and organelle movement, cell signaling, and the establishment and maintenance of cell junctions and cell shape.

Myosin, a large family of motor proteins found in eukaryotic tissues, is a thick filament-based molecule that harnesses the free energy furnished by ATP hydrolysis to perform mechanical work against actin proteins of the thin filament. The cyclic attachment and detachment of myosin with actin that generates muscle force and shortening is $\mathrm{Ca}^{2+}$ regulated and is responsible for actin-based motility (Vandenboom R. 2004).

MHC is a structurally bound contractile protein of the thick filaments, which has a ATPase function that could involve binding with actin. In this work, MHC and actin were found to have decreased in skeletal muscle. It is probable that during intense activation of skeletal muscle, contractile function becomes impaired, and attachment of myosin with actin, which generates muscle force, was no longer needed.
A

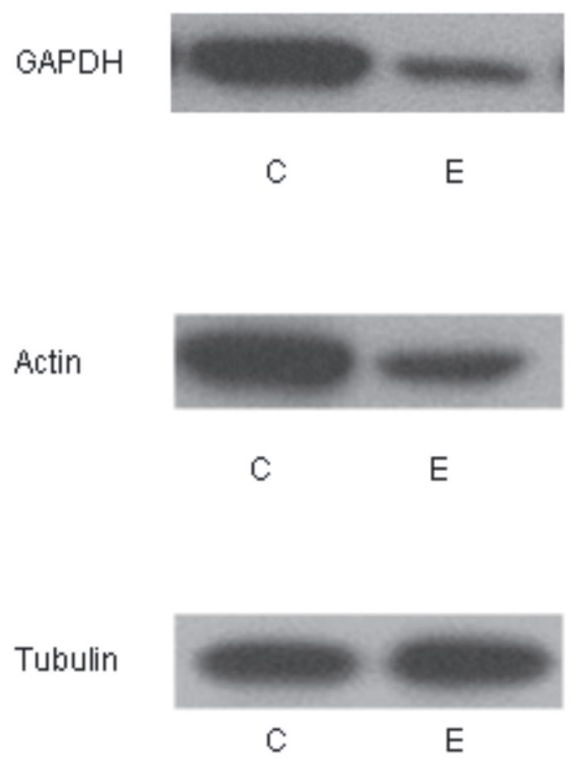

B
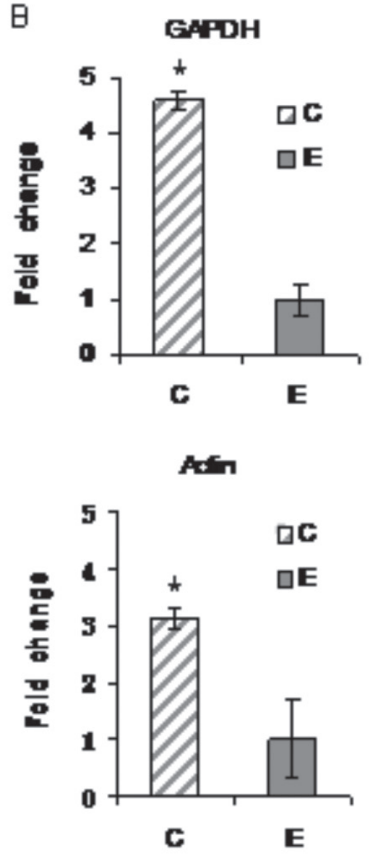

Fig. 4: (A) Westem blots showing expression of GAPDH an actin among control and treated groups. Equal loading of the samples was evaluated by reprobingmthe menbranes with tubuin antiboby. (B)Quantitative fold change. *indicates significant difference, $\mathrm{p}<0,05$. 
In contrast to MHC, there are only three TnI isoforms, one for slow-twitch skeletal muscle, one for fast-twitch skeletal muscle, and one for myocardium (North KN et al. 2008). Our results provide evidence that the high muscle force associated with eccentric contraction causes rapid removal of fsTnI. These findings support the concept that eccentric exercise initiates a series of events that result in rapid disruption of the cytoskeletal network and contractile apparatus, which could be the mechanism for deterioration of the contractile response and loss in force generation.

Skeletal muscle is the most important effector of exerciseinduced fatigue, although several proteins have been found to be associated. However, their role in exercise-induced fatigue is not well understood. Therefore, further investigation of their role will help to elucidate the mechanism of exercise induced fatigue.

\section{REFERENCES}

ADAM J ROSE and ERIC A (2009) Richter Regulatory mechanisms of skeletal muscle protein turnover during exercisen J Appl Physiol, 106: 1702 - 1711.

ALAN H and MICHAEL J MCKENNA (2009) Dissociation between force and maximal $\mathrm{Na}+, \mathrm{K}+$-ATPase activity in rat fast-twitch skeletal muscle with fatiguing in vitro stimulation Craig A Goodman, , 105 (4), 575-583.

ANDERS J, DAHL STEDT, ABRAM KATZ, HÅKAN WESTERBLAD (2000) Is creatine kinase responsible for fatigue? Studies of isolated skeletal muscle deficient in creatine kinase. The FASEB Journa14:982-990.

BORJA G, AMELIA GG, TERESA F, et al. (2010) SIRT 1, AMP-activated protein kinase phosphorylation and downstream kinases in response to a single bout of sprint exercise: influence of glucose ingestion, 109, 4, 731-743.

BURNISTON JG (2008) Changes in the rat skeletal muscle proteome induced by moderate-intensity endurance exercise. Biochimica Biophysica Acta 1784:1077-1086.

CHOSA E, SEKIMOTO T, SONODA N, YAMAMOTO K, MATSUDA H, TAKAHAMA K, TAJIMA N (2003) Evaluation of human beta-enolase as a serum marker for exercise-induced muscle damage.Clin J Sport Med. 13(4):209-12.

CHUANG DM, HOUGH C, SENATOROV VV (2005) Glyceraldehyde-3phosphate dehydrogenase, apoptosis, and neurodegenerative diseases. Annu. Rev. Pharmacol. Toxicol. 45:269-90.

GORG A, POSTEL V, GUNTHER S (1988) The current state of twodimensional electrophoresis with immobilized $\mathrm{pH}$ gradients Electrophoresis 9:531-546.
GANDEVIA SC (2001) Spinal and Supraspinal Factors in Human Muscle Fatigue. Physiol. Rev. 81: 1725-1789.

HITTEL DS, HATHOUT Y, HOFFMAN EP (2007) Proteomics and systems biology in exercise and sport sciences research, Exerc. Sport Sci. Rev. 35,5-11.

HOU L, LIU X, QIAO D (2005) The Establishment of Exercise-induced Fatigue Model in Rats. Laborarory Animal Science and Management. 22(1):1-3

KRUGER NJ, WALKER JM (1996) The Protein Protocols Handbook. Totowa NJ: Human Press:15-20.

KELLER A, OTT MO, LAMANDE N, LUCAS M, GROS F, BUCKINGHAM M and LAZAR M (1992) Mech Dev 38:41-54.

LABELLA JJ, DAOOD MJ, KORETSKY AP, ROMAN BB, SIECK GC (1998) Absence of myofibrillar creatine kinase and diaphragm isometric function during repetitive activation. J Appl Physiol 84:1166-1173.

MOMKEN I, LECHENE P, KOULMANN N, FORTIN D, MATEO P, DOAN BT, HOERTER J, BIGARD X, VEKSLER V, VENTURA-CLAPIER R (2005) Impaired voluntary running capacity of creatine kinase-deficient mice. J Physiol. 15;565 (Pt 3):951-64.

MICHAEL A, SIROVER (1999) New insights into an old protein: the functional diversity of mammalian glyceraldehyde 3 phosphate dehydrogenase. Biochim Biophys Acta , 1432: 159-184.

NORTH KN, LAING Skeletal muscle alpha-actin diseases. Adv Exp Med Biol. (2008); 642:15-27.

POWERS SK, JACKSON MJ (2008) Exercise-induced oxidative stress: cellular mechanisms and impact on muscle force production. Physiol Rev. 88(4):1243-76.

REID MB (2008) Free radicals and muscle fatigue: Of ROS, canaries, and the IOC. Free Radic Biol Med. 15;44(2):169-79.

STEEGHS KA, BENDERS A, OERLEMANS F, HAAN A, HEERSCHAP A, RUITENBEEK W, JOST C, VAN DI, PERRYMAN B, PETTE D, BRUCKWILDER M, KOUDIJS J, JAP P, and WIERINGA B (1997) Altered Ca21 responses in muscles with combined mitochondrial and cytosolic creatine kinase deficiencies. Cell 89: 93-103.

TERADA S, YOKOZEKI T, KAWANAKA K, OGAWA K, HIGUCHI M, EZAKI $O$, and TABATA I. Effects of high-intensity swimming training on GLUT-4 and glucose transport activity in rat skeletal muscle. J Appl Physiol 90: 2019-2024, (2001).

VANDENBOOM R (2004)The myofibrillar complex and fatigue:a review.Can Applied Physiology, 29(3):330-56.

WESTERBLAD H, ALLEN DG, BRUTON JD, ANDRADE FH, LANNERGREN J (1998). Mechanisms underlying the reduction of isometric force in skeletal muscle fatigue. Acta Physio Scand 162:253-260.

WATCHKO JF, DAOOD MJ, SIECK GC, LABELLA JJ, AMEREDES BT, KORETSKY AP, and WIERINGA B (1997) Combined myofibrillar and mitochondrial creatine kinase deficiency impairs mouse diaphragm isotonic function. J Appl Physiol 82: 1416-1423.

YAMAGUCHI W, FUJIMOTO E, HIGUCHI M, TABATA I (2010) A DIGE proteomic analysis for high-intensity exercise-trained rat skeletal muscle. J Biochem. 148 (3):327-33. 